\title{
Units and norm residue symbol
}

\author{
by \\ Bruno Anglès (Caen)
}

Let $p$ be an odd prime number, $p \geq 5$. Let $\zeta_{p}$ be a primitive $p$ th root of unity and consider the following equation:

(*) $\quad a, b \in \mathbb{Z}, a b \neq 0, \operatorname{gcd}(a, b)=1,\left(a-b \zeta_{p}\right) \mathbb{Z}\left[\zeta_{p}\right]=I^{p}, I$ ideal of $\mathbb{Z}\left[\zeta_{p}\right]$.

Then one can show that the $A B C$ conjecture implies that the above equation has a finite number of solutions, and, if $p$ is large enough, $(*)$ has only the trivial solutions, i.e. $a=1, b=-1$, and $a=-1, b=1$.

When studying the first case of $(*)($ i.e. $a b(a+b) \not \equiv 0(\bmod p))$, G. Terjanian was led to conjecture that the Kummer system of congruences has only the trivial solutions (see [8] and Section 5). In this paper we prove that Eichler's Theorem applies to Terjanian's conjecture (Corollary 5.5). More precisely, we prove that if $i(p)<\sqrt{p}-2$ then Terjanian's conjecture is true for the prime $p$, where $i(p)$ is the index of irregularity of $p$.

Let $F$ be a real subfield of $\mathbb{Q}\left(\zeta_{p}\right)$ and let $E_{F}$ be the group of units of $F$. Our aim is to study the Kummer subgroup of $E_{F}$ :

$$
E_{F}^{\mathrm{Kum}}=\left\{\varepsilon \in E_{F}: \exists a \in \mathbb{Z}, \varepsilon \equiv a(\bmod p)\right\} .
$$

We show that there exists a duality between $E_{F} / E_{F}^{\mathrm{Kum}}$ and the orthogonal of $E_{F}$ for the norm residue symbol (see Theorem 4.4). A natural problem arises: do we have an equivalence in Kummer's Lemma (see Section 3)? We show that this question is connected to a class number congruence obtained by T. Metsänkylä (see [4] and Section 6). In particular, we are led to investigate the orthogonal of the group of units of $\mathbb{Q}\left(\zeta_{p}\right)$ for the norm residue symbol and, thus, this leads us to Terjanian's conjecture.

Finally, we would like to mention the following question which we call the "weak Kummer-Vandiver conjecture": let $E$ be the group of units of $\mathbb{Q}\left(\zeta_{p}\right)$ and let $C$ be the group of cyclotomic units of $\mathbb{Q}\left(\zeta_{p}\right)$; do we have $E^{\perp}=C^{\perp}$ (see Section 4)?

2000 Mathematics Subject Classification: 11R18, 11S31. 
1. Notations. Let $p$ be an odd prime number. Let $\mathbb{Z}_{p}$ be the ring of $p$-adic integers, $\mathbb{Q}_{p}$ the field of $p$-adic numbers, and $\mathbb{C}_{p}$ a completion of an algebraic closure of $\mathbb{Q}_{p}$. All the finite extensions of $\mathbb{Q}_{p}$ considered in this paper are contained in $\mathbb{C}_{p}$.

Let $L / \mathbb{Q}_{p}$ be a finite extension. We set:

- $O_{L}$ - the integral closure of $\mathbb{Z}_{p}$ in $L$,

- $\mathfrak{p}_{L}$ - the maximal ideal of $O_{L}$,

- $v_{L}$ - the normalized discrete valuation on $L$ associated with $\mathfrak{p}_{L}$,

- $U_{L}$ - the group of units of $O_{L}$ and for $n \geq 1, U_{L}^{(n)}=1+\mathfrak{p}_{L}^{n}$.

Let $L / \mathbb{Q}_{p}$ be a finite extension and let $L^{\prime} / L$ be a finite abelian extension. We denote the local Artin map associated with $L^{\prime} / L$ by $\left(\cdot, L^{\prime} / L\right)$.

Let $\zeta_{p}$ be a fixed primitive $p$ th root of unity in $\mathbb{C}_{p}$. We set $\lambda_{p}=\zeta_{p}-1$ and $K=\mathbb{Q}_{p}\left(\zeta_{p}\right)$. For $\alpha, \beta \in K^{*}$, we define the norm residue symbol $(\alpha, \beta)$ as follows:

$$
(\alpha, \beta)=\frac{(\beta, K(\gamma) / K)(\gamma)}{\gamma}
$$

where $\gamma \in \mathbb{C}_{p}$ is such that $\gamma^{p}=\alpha$.

Let $G=\operatorname{Gal}\left(K / \mathbb{Q}_{p}\right)$. For $a \in \mathbb{Z} \backslash p \mathbb{Z}$ we define $\sigma_{a}$ to be the element of $G$ such that $\sigma_{a}\left(\zeta_{p}\right)=\zeta_{p}^{a}$. Recall that we have an isomorphism of groups $(\mathbb{Z} / p \mathbb{Z})^{*} \rightarrow G, \bar{a} \mapsto \sigma_{a}$. Let $\widehat{G}$ be the set of group homomorphisms between $G$ and $\mathbb{Z}_{p}^{*}$. The Teichmüller character $\omega$ is the element $\omega \in \widehat{G}$ such that

$$
\omega\left(\sigma_{a}\right) \equiv a(\bmod p) .
$$

Recall that $\widehat{G}$ is a cyclic group and that $\omega$ is a generator of $\widehat{G}$.

We view $\mathbb{Q}$ as contained in $\mathbb{Q}_{p}$. Let $F / \mathbb{Q}$ be a finite extension, $F \subset \mathbb{C}_{p}$. We set

- $\widehat{F}=F \mathbb{Q}_{p}$,

- $O_{F}$ - the ring of integers of $F$,

- $E_{F}$ - the group of units of $O_{F}$,

- $\mathfrak{p}_{F}=\mathfrak{p}_{\hat{F}} \cap O_{F}$,

- $h_{F}$ — the class number of $F$.

If $A$ is a commutative unitary ring, we denote the set of invertible elements of $A$ by $A^{*}$. Let $n \geq 1$ be an integer. We denote the group of $n$th roots of unity in $\mathbb{C}_{p}$ by $\mu_{n}$.

2. Some results from Lubin-Tate theory. First, we recall some basic facts from Lubin-Tate theory (see [3], Chapter 8). We consider the following two elements in $\mathbb{Z}_{p}[[X]]$ :

$$
T(X)=(1+X)^{p}-1 \text { and } L(X)=X^{p}+p X .
$$


Then $T$ and $L$ are Lubin-Tate polynomials. Thus there exist two formal groups $F_{T}=\mathbb{G}_{\mathrm{m}}$ and $F_{L}$ in $\mathbb{Z}_{p}[[X, Y]]$ such that

$$
T \circ F_{T}=F_{T} \circ T \text { and } L \circ F_{L}=F_{L} \circ L .
$$

We have two ring homomorphisms: $\mathbb{Z}_{p} \rightarrow$ End $_{\mathbb{Z}_{p}} \mathbb{G}_{\mathrm{m}}, a \mapsto[a]_{T}=(1+X)^{a}-1$ and $\mathbb{Z}_{p} \rightarrow \operatorname{End}_{\mathbb{Z}_{p}} F_{L}, a \mapsto[a]_{L}$. Note that

- $\forall a \in \mathbb{Z}_{p},[a]_{T} \equiv[a]_{L} \equiv a X(\bmod \operatorname{deg} 2)$,

- $F_{T}(X, Y)=(1+X)(1+Y)-1, F_{L}(X, Y) \equiv X+Y(\bmod \operatorname{deg} p)$,

- $\forall a \in \mathbb{Z}_{p},[a]_{L} \equiv a X(\bmod \operatorname{deg} p), \forall \varepsilon \in \mu_{p-1},[\varepsilon]_{L}=\varepsilon X$.

We set

$$
\begin{aligned}
& \log _{T}(X)=\lim _{n \geq 1} \frac{1}{p^{n}}\left[p^{n}\right]_{T} \in \mathbb{Q}_{p}[[X]], \\
& \log _{L}(X)=\lim _{n \geq 1} \frac{1}{p^{n}}\left[p^{n}\right]_{L} \in \mathbb{Q}_{p}[[X]] .
\end{aligned}
$$

Note that

$$
\log _{T}(X)=\sum_{n \geq 1}(-1)^{n+1} \frac{X^{n}}{n} \quad \text { and } \quad \log _{L}(X) \equiv X(\bmod \operatorname{deg} p) .
$$

We denote the inverses of $\log _{T}$ and $\log _{L}$ by $\operatorname{Exp}_{T}$ and $\operatorname{Exp}_{L}$ respectively.

We set $f_{p}(X)=\operatorname{Exp}_{T} \circ \log _{L}$ and $g_{p}(X)=\operatorname{Exp}_{L} \circ \log _{T}$. Then $f_{p}$ and $g_{p}$ are elements of $\mathbb{Z}_{p}[[X]]$ and we have:

- $f_{p}(X) \equiv g_{p}(X) \equiv X(\bmod \operatorname{deg} 2)$,

- $\forall a \in \mathbb{Z}_{p}, f_{p} \circ[a]_{L}=[a]_{T} \circ f_{p}$ and $g_{p} \circ[a]_{T}=[a]_{L} \circ g_{p}$,

- $f_{p} \circ F_{L}=F_{T} \circ f_{p}$ and $g_{p} \circ F_{T}=F_{L} \circ g_{p}$,

- $f_{p} \circ g_{p}=g_{p} \circ f_{p}=X$.

Let $v_{p}$ be the $p$-adic valuation on $\mathbb{C}_{p}$ such that $v_{p}(p)=1$. Set $D=$ $\left\{\alpha \in \mathbb{C}_{p}: v_{p}(\alpha)>0\right\}$. Then $T$ induces a new structure of $\mathbb{Z}_{p}$-module for $D$ and we denote this $\mathbb{Z}_{p}$-module by $D_{T}$; the same holds for $L$ and we denote $D$ equipped with the structure of $\mathbb{Z}_{p}$-module induced by $L$ by $D_{L}$. We have an isomorphism of $\mathbb{Z}_{p}$-modules $D_{T} \rightarrow D_{L}, \alpha \mapsto g_{p}(\alpha)$. Set $\Lambda_{T}=\left\{\alpha \in \mathbb{C}_{p}:[p]_{T}(\alpha)=0\right\}$ and $\Lambda_{L}=\left\{\alpha \in \mathbb{C}_{p}:[p]_{L}(\alpha)=0\right\}$. Then $\Lambda_{T}$ is a $\mathbb{Z}_{p}$-submodule of $D_{T}$ and $\Lambda_{L}$ is a $\mathbb{Z}_{p}$-submodule of $D_{L}$. Note that $g_{p}$ induces an isomorphism of the $\mathbb{Z}_{p}$-modules $\Lambda_{T}$ and $\Lambda_{L}$. We have $\lambda_{p} \in \Lambda_{T}$. We set

$$
\lambda_{L}=g_{p}\left(\lambda_{p}\right) .
$$

Note that $\lambda_{L}^{p-1}=-p$ and $K=\mathbb{Q}_{p}\left(\lambda_{p}\right)=\mathbb{Q}_{p}\left(\lambda_{L}\right)$.

Lemma 2.1. We have

$$
g_{p}(X) \equiv \sum_{n=1}^{p-1}(-1)^{n+1} \frac{X^{n}}{n}\left(\bmod X^{p} \mathbb{Z}_{p}[[X]]\right),
$$




$$
f_{p}(X) \equiv \sum_{n=1}^{p-1} \frac{X^{n}}{n !}\left(\bmod X^{p} \mathbb{Z}_{p}[[X]]\right) .
$$

Proof. This comes from the fact that $\operatorname{Exp}_{L}(X) \equiv \log _{L}(X) \equiv X$ $(\bmod \operatorname{deg} p)$.

Corollary 2.2 .

(i) $\lambda_{L} \equiv \sum_{n=1}^{p-1}(-1)^{n+1} \frac{\lambda_{p}^{n}}{n}\left(\bmod \mathfrak{p}_{K}^{p}\right)$;

(ii) $\lambda_{p} \equiv \sum_{n=1}^{p-1} \frac{\lambda_{L}^{n}}{n !}\left(\bmod \mathfrak{p}_{K}^{p}\right)$.

Lemma 2.3. Let $\sigma \in G$.

(i) $\sigma\left(\lambda_{p}\right)=[\omega(\sigma)]_{T}\left(\lambda_{p}\right)$;

(ii) $\sigma\left(\lambda_{L}\right)=\omega(\sigma) \lambda_{L}$.

Proof. The first assertion is obvious. We have

$$
\sigma\left(\lambda_{L}\right)=\sigma\left(g_{p}\left(\lambda_{p}\right)\right)=g_{p}\left(\sigma\left(\lambda_{p}\right)\right) .
$$

Thus $\sigma\left(\lambda_{L}\right)=g_{p}\left([\omega(\sigma)]_{T}\left(\lambda_{p}\right)\right)=[\omega(\sigma)]_{L}\left(g_{p}\left(\lambda_{p}\right)\right)=\omega(\sigma) \lambda_{L}$.

Let $k$ be an integer, $1 \leq k \leq p-1$. We set

$$
\eta_{k}=\sum_{i=1}^{p-1}(i !)^{k-1} \tau\left(\omega^{-i}\right)^{k}
$$

where, for $i=1, \ldots, p-1$,

$$
\tau\left(\omega^{-i}\right)=-\sum_{\sigma \in G} \omega(\sigma)^{-i} \sigma\left(\lambda_{p}\right) \in \mathfrak{p}_{K}
$$

Note that $\eta_{1}=(1-p) \lambda_{p}$.

Proposition 2.4. Let $k$ be an integer, $1 \leq k \leq p-1$.

(i) $\eta_{k} \equiv f_{p}\left(\lambda_{L}^{k}\right)\left(\bmod \mathfrak{p}_{K}^{p}\right)$;

(ii) $\lambda_{L}^{k} \equiv g_{p}\left(\eta_{k}\right)\left(\bmod \mathfrak{p}_{K}^{p}\right)$;

(iii) $\forall \sigma \in G, \sigma\left(1+\eta_{k}\right) \equiv\left(1+\eta_{k}\right)^{\omega(\sigma)^{k}}\left(\bmod \mathfrak{p}_{K}^{p}\right)$.

Proof. Let $\sigma \in G$. We have

$$
\sigma\left(\lambda_{p}\right) \equiv \sum_{n=1}^{p-1} \omega(\sigma)^{n} \frac{\lambda_{L}^{n}}{n !}\left(\bmod \mathfrak{p}_{K}^{p}\right) .
$$

Thus

$$
\tau\left(\omega^{-i}\right) \equiv \frac{\lambda_{L}^{i}}{i !}\left(\bmod \mathfrak{p}_{K}^{p}\right) .
$$

Therefore we have (i) and (ii). Now, let $\sigma \in G$. Then 


$$
\sigma\left(\eta_{k}\right) \equiv f_{p}\left(\omega(\sigma)^{k} \lambda_{L}^{k}\right) \equiv\left[\omega(\sigma)^{k}\right]_{T}\left(f_{p}\left(\lambda_{L}^{k}\right)\right) \equiv\left(1+\eta_{k}\right)^{\omega(\sigma)^{k}}-1\left(\bmod \mathfrak{p}_{K}^{p}\right) .
$$

Thus we have (iii).

Now, we recall the definition of the Kummer homomorphisms (see [3], Chapter 7). Let $u \in U_{K}$ and write $u=h\left(\lambda_{L}\right)$ for some $h(X) \in \mathbb{Z}_{p}[[X]]$. Then $h^{\prime}\left(\lambda_{L}\right) / u$ is well defined modulo $\mathfrak{p}_{K}^{p-2}$ and we can write

$$
\frac{h^{\prime}\left(\lambda_{L}\right)}{u} \equiv \sum_{k=1}^{p-2} \varphi_{k}(u) \lambda_{L}^{k-1}\left(\bmod \mathfrak{p}_{K}^{p-2}\right)
$$

where $\varphi_{k}(u)$ is in $\mathbb{Z}_{p}$ modulo $p \mathbb{Z}_{p}$ for $k=1, \ldots, p-2$. The map $\varphi_{k}$ is called the Kummer homomorphism of degree $k$.

We have the following basic properties:

- $\varphi_{k}: U_{K} \rightarrow \mathbb{F}_{p}$ is a surjective group homomorphism and $\mu_{p-1} U_{K}^{(k+1)} \subset$ $\operatorname{ker} \varphi_{k}$;

- $\forall \sigma \in G, \forall u \in U_{K}, \varphi_{k}(\sigma(u)) \equiv \omega(\sigma)^{k} \varphi_{k}(u)(\bmod p)$;

- $\forall u \in U_{K}^{(1)}, \forall a \in \mathbb{Z}_{p}, \varphi_{k}\left(u^{a}\right) \equiv a \varphi_{k}(u)(\bmod p)$;

- $\bigcap_{1 \leq k \leq p-2} \operatorname{ker} \varphi_{k}=\mu_{p-1} U_{K}^{(p-1)}$.

We calculate the values of these homomorphisms for some remarkable elements.

Proposition 2.5.

(i) $\varphi_{1}\left(\zeta_{p}\right)=1$ and for $k \geq 2, \varphi_{k}\left(\zeta_{p}\right)=0$;

(ii) $\varphi_{k}\left(\lambda_{p} / \lambda_{L}\right)=(-1)^{k} B_{k} / k$ !, where $B_{k}$ is the $k$ th Bernoulli number;

(iii) let $\sigma \in G, \varphi_{k}\left(\sigma\left(\lambda_{p}\right) / \lambda_{p}\right)=(-1)^{k}\left(\omega(\sigma)^{k}-1\right) B_{k} / k !$;

(iv) $\varphi_{k}\left(1+\eta_{i}\right)=0$ if $k \neq i$ and $\varphi_{k}\left(1+\eta_{k}\right)=k$;

(v) let $a \in \mathbb{Z}, a \neq \equiv 1(\bmod p), \varphi_{1}\left(a-\zeta_{p}\right)=-1 /(a-1)$ and for $k \geq 2$,

$$
\varphi_{k}\left(a-\zeta_{p}\right)=\frac{(-1)^{k-1}}{(k-1) !(a-1)} M_{k}(a),
$$

where $M_{k}(X)=\sum_{i=1}^{p-1} i^{k-1} X^{i}$ is the kth Mirimanoff polynomial.

Proof. (i) Write $h(X)=\sum_{n=0}^{p-2} X^{n} / n$ !. Then $\zeta_{p} \equiv h\left(\lambda_{L}\right)\left(\bmod \mathfrak{p}_{K}^{p}\right)$. Thus $\varphi_{k}\left(\zeta_{p}\right)=\varphi_{k}\left(h\left(\lambda_{L}\right)\right)$. But

$$
\frac{h^{\prime}\left(\lambda_{L}\right)}{h\left(\lambda_{L}\right)} \equiv \zeta_{p}^{-1} h^{\prime}\left(\lambda_{L}\right) \equiv\left(\sum_{n=0}^{p-3}(-1)^{n} \frac{\lambda_{L}^{n}}{n !}\right)\left(\sum_{n=0}^{p-3} \frac{\lambda_{L}^{n}}{n !}\right) \equiv 1\left(\bmod \mathfrak{p}_{K}^{p-2}\right) .
$$

(ii) Put $h(X)=f_{p}(X) / X$. Then $\lambda_{p} / \lambda_{L}=h\left(\lambda_{L}\right)$. One can show that

$$
\frac{h^{\prime}(X)}{h(X)} \equiv B_{1}+1+\sum_{k \geq 2} \frac{B_{k}}{k !} X^{k-1}(\bmod \operatorname{deg} p-2) \text {. }
$$

The result follows. 
(iii) Let $\sigma \in G$. We have

$\varphi_{k}\left(\frac{\sigma\left(\lambda_{p}\right)}{\lambda_{p}}\right)=\varphi_{k}\left(\sigma\left(\frac{\lambda_{p}}{\lambda_{L}}\right)\right)+\varphi_{k}\left(\frac{\sigma\left(\lambda_{L}\right)}{\lambda_{p}}\right)=\left(\omega(\sigma)^{k}-1\right) \varphi_{k}\left(\frac{\lambda_{p}}{\lambda_{L}}\right)$.

(iv) Set $h(X)=f_{p}\left(X^{k}\right)+1$. We have $1+\eta_{k} \equiv h\left(\lambda_{L}\right)\left(\bmod \mathfrak{p}_{K}^{p}\right)$. Therefore $\varphi_{i}\left(1+\eta_{k}\right)=\varphi_{i}\left(h\left(\lambda_{L}\right)\right)$. But

$$
\frac{h^{\prime}(X)}{h(X)} \equiv k X^{k-1}(\bmod \operatorname{deg} p-2),
$$

and the result follows.

(v) We have

$$
a-\zeta_{p} \equiv a-1-\lambda_{L}\left(\bmod \mathfrak{p}_{K}^{2}\right)
$$

Therefore

$$
\varphi_{1}\left(a-\zeta_{p}\right)=\varphi_{1}\left(a-1-\lambda_{L}\right)=\frac{-1}{a-1} .
$$

If $a \equiv 0(\bmod p)$, then for $k \geq 2$, we have $\varphi_{k}\left(a-\zeta_{p}\right)=0$. Now, we suppose that $a \neq \equiv(\bmod p)$. We have

$$
D^{k} \log (a-\operatorname{Exp}(X))_{X=0} \equiv(k-1) ! \varphi_{k}\left(a-\zeta_{p}\right)(\bmod p) .
$$

But, by [5], Chapter VIII,

$$
D^{k} \log (a-\operatorname{Exp}(X))_{X=0} \equiv \frac{(-1)^{p-k}}{a-1} M_{k}(a)(\bmod p)
$$

The result follows.

We recall some basic facts about $\mathbb{F}_{p}[G]$-modules. For $\chi \in \widehat{G}$, we write

$$
e_{\chi}=\frac{1}{p-1} \sum_{\sigma \in G} \chi(\sigma) \sigma^{-1}(\bmod p) \text {. }
$$

We have

- $e_{\chi}^{2}=e_{\chi}$

- $e_{\chi} e_{\psi}=0$ if $\chi \neq \psi$;

- $1=\sum_{\chi \in \widehat{G}} e_{\chi}$;

- $\forall \sigma \in G, \sigma e_{\chi}=\chi(\sigma) e_{\chi}$.

Let $A$ be an $\mathbb{F}_{p}[G]$-module. For $1 \leq i \leq p-1$, we set

$$
A(i)=e_{\omega^{i}} A=\left\{a \in A: \forall \sigma \in G, \sigma(a)=\omega(\sigma)^{i} a\right\} .
$$

We have

$$
A=\bigoplus_{i=1}^{p-1} A(i)
$$

We set

$$
\mathcal{U}=\frac{U_{K}}{\mu_{p-1} U_{K}^{(p)}}
$$


It is clear that $\mathcal{U}$ is a finite $\mathbb{F}_{p}[G]$-module and that, for $1 \leq i \leq p-1, \mathcal{U}(i)$ is an $\mathbb{F}_{p}$-vector space of dimension 1 . More precisely, let $u \in \mathcal{U}$; then $e_{\omega^{i}} u$ generates $\mathcal{U}(i)$ if and only if

- $\varphi_{i}(u) \neq 0$ if $1 \leq i \leq p-2$

- $N_{K / \mathbb{Q}_{p}}(u) \not \equiv 1\left(\bmod p^{2}\right)$ for $i=p-1$.

In particular, for $1 \leq k \leq p-1,1+\eta_{k} \in \mathcal{U}(k)$ and $1+\eta_{k}$ generates $\mathcal{U}(k)$.

Proposition 2.6. Let $u \in U_{K}$. Then

$$
\log _{p}(u) \equiv \frac{N_{K / \mathbb{Q}_{p}}(u)-1}{p} \lambda_{L}^{p-1}+\sum_{k=2}^{p-2} \frac{1}{k} \varphi_{k}(u) \lambda_{L}^{k}\left(\bmod \mathfrak{p}_{K}^{p}\right),
$$

where $\log _{p}$ is the usual $p$-adic logarithm on $\mathbb{C}_{p}^{*}$.

Proof. Note that we can suppose $u \in U_{K}^{(1)}$. We have $\log _{p}(u) \in \mathfrak{p}_{K}$ and, if $u \in U_{K}^{(p)}, \log _{p}(u) \in \mathfrak{p}_{K}^{p}$. Therefore, $\log _{p}$ induces a group homomorphism between $\mathcal{U}$ and $\mathfrak{p}_{k} / \mathfrak{p}_{K}^{p}$. Note that, for $k \geq 2$,

$$
\log _{p}\left(1+\eta_{k}\right) \equiv g_{p}\left(\eta_{k}\right) \equiv \lambda_{L}^{k}\left(\bmod \mathfrak{p}_{K}^{p}\right)
$$

and

$$
\log _{p}\left(1+\eta_{1}\right) \equiv \log _{p}\left(\zeta_{p}\right) \equiv 0\left(\bmod \mathfrak{p}_{K}^{p}\right) .
$$

Let $u \in U_{K}^{(2)}$. We have

$$
u \equiv \prod_{k=2}^{p-1}\left(1+\eta_{k}\right)^{a_{k}}\left(\bmod U_{K}^{(p)}\right)
$$

where $a_{k} \in \mathbb{F}_{p}$. Thus

$$
\log _{p}(u) \equiv \sum_{k=2}^{p-1} a_{k} \lambda_{L}^{k} \equiv \sum_{k=2}^{p-2} \frac{1}{k} \varphi_{k}(u) \lambda_{L}^{k}+a_{p-1} \lambda_{L}^{p-1}\left(\bmod \mathfrak{p}_{K}^{p}\right) .
$$

But

$$
e_{\omega^{p-1}} u \equiv\left(1+\eta_{p-1}\right)^{a_{p-1}} \equiv N_{K / \mathbb{Q}_{p}}(u)^{-1}\left(\bmod U_{K}^{(p)}\right) .
$$

Thus

$$
-\log _{p}\left(N_{K / \mathbb{Q}_{p}}(u)\right) \equiv-a_{p-1} p\left(\bmod \mathfrak{p}_{K}^{p}\right)
$$

But

$$
\log _{p}\left(N_{K / \mathbb{Q}_{p}}(u)\right) \equiv N_{K / \mathbb{Q}_{p}}(u)-1\left(\bmod p^{2}\right) .
$$

Therefore we get our result for $u \in U_{K}^{(2)}$.

Now, if $u \in U_{K}^{(1)}$, there exists an integer $a_{1}$ such that $u\left(1+\eta_{1}\right)^{a_{1}} \in U_{K}^{(2)}$. But

$$
\begin{aligned}
\log _{p}\left(u\left(1+\eta_{1}\right)^{a_{1}}\right) & \equiv \log _{p}(u)\left(\bmod \mathfrak{p}_{K}^{p}\right), \\
N_{K / \mathbb{Q}_{p}}\left(u\left(1+\eta_{1}\right)^{a_{1}}\right) & \equiv N_{K / \mathbb{Q}_{p}}(u)\left(\bmod p^{2}\right) .
\end{aligned}
$$


For $k \geq 2$,

$$
\varphi_{k}\left(u\left(1+\eta_{1}\right)^{a_{1}}\right)=\varphi_{k}(u) .
$$

The proposition follows.

We recall the definition of the local Kummer symbol relative to $L$ (see [3], Chapter 8). Let $z \in \mathfrak{p}_{K}$ and let $\alpha \in K^{*}$. Let $t \in \mathbb{C}_{p}$ be such that $[p]_{L}(t)=z$. We set

$$
\langle z, \alpha\rangle_{L}=F_{L}((\alpha, K(t) / K)(t),-t) \in \Lambda_{L} .
$$

This symbol is connected to the norm residue symbol as follows: let $u \in U_{K}^{(1)}$ and let $\alpha \in K^{*}$; then

$$
(u, \alpha)-1=f_{p}\left(\left\langle g_{p}(u-1), \alpha\right\rangle_{L}\right) .
$$

Furthermore, we have the following explicit reciprocity law for $\langle\cdot, \cdot\rangle_{L}$ :

Theorem 2.7. Let $z \in \mathfrak{p}_{K}$ and let $u \in U_{K}$. Write $z \equiv \sum_{i=1}^{p-1} a_{i} \lambda_{L}^{i}$ $\left(\bmod \mathfrak{p}_{K}^{p}\right)$, where $a_{i} \in \mathbb{F}_{p}$. Then

$$
\langle z, u\rangle_{L}=\left[a_{1} \frac{N_{K / \mathbb{Q}_{p}}\left(u^{-1}\right)-1}{p}+\sum_{i=2}^{p-1} a_{i} \varphi_{p-i}(u)\right]_{L}\left(\lambda_{L}\right) .
$$

Proof. See [3], Chapter 9.

3. Kummer subgroups of units. Recall that $\mathcal{U}=U_{K} /\left(\mu_{p-1} U_{K}^{(p)}\right)$. Set

$$
V=\mathbb{Q}\left(\zeta_{p}\right) \cap U_{K}, \quad V^{\mathrm{Kum}}=V \cap \mu_{p-1} U_{K}^{(p)}, \quad \mathcal{V}=V / V^{\mathrm{Kum}} .
$$

Then we have an isomorphism of the $\mathbb{F}_{p}[G]$-modules $\mathcal{V}$ and $\mathcal{U}$.

Let $B$ be a subgroup of $V$. We define the Kummer subgroup of $B$ to be

$$
B^{\mathrm{Kum}}=B \cap V^{\mathrm{Kum}}=B \cap \mu_{p-1} U_{K}^{(p)} .
$$

Note that

$$
B^{\mathrm{Kum}} \subset\left\{\alpha \in B: \exists a \in \mathbb{Z}, \alpha \equiv a\left(\bmod \mathfrak{p}_{K}^{p}\right)\right\} .
$$

Let $F$ be a real subfield of $\mathbb{Q}\left(\zeta_{p}\right)$. The group of cyclotomic units of $F$ is the subgroup of $E_{F}$ generated by -1 and $N_{\mathbb{Q}\left(\zeta_{p}\right)^{+} / F}\left(\zeta_{p}^{(1-a) / 2}\left(\zeta_{p}^{a}-1\right) /\left(\zeta_{p}-1\right)\right)$, for $2 \leq a \leq(p-1) / 2$; we denote this group by $\mathrm{Cyc}_{F}$. Recall that

$$
\left(E_{F}: \mathrm{Cyc}_{F}\right)=h_{F} .
$$

In this section, our aim is to study the $\mathbb{F}_{p}[G]$-module $\mathrm{Cyc}_{F} / \mathrm{Cyc}_{F}^{\mathrm{Kum}}$. In particular, Theorem 3.2 will generalize a result of Vostokov (see [9], Theorem 1) and we will obtain Kummer's Lemma (see [10], Theorem 5.36) as a corollary.

Now, let $F$ be a real subfield of $\mathbb{Q}\left(\zeta_{p}\right)$ and set $l=[F: \mathbb{Q}]$. We suppose that $l \geq 2$. 
LEMMA 3.1. We have

$$
\begin{aligned}
& E_{F}^{\mathrm{Kum}}=\left\{\alpha \in E_{F}: \exists a \in \mathbb{Z}, \alpha \equiv a(\bmod p)\right\}=E_{F} \cap\left(K^{*}\right)^{p}, \\
& E_{F}^{\mathrm{Kum}}=\left\{\alpha \in E_{F}: \log _{p}(\alpha) \equiv 0\left(\bmod \mathfrak{p}_{K}^{p}\right)\right\} .
\end{aligned}
$$

Proof. By [10], page 80,

$$
\left\{\alpha \in E_{F}: \exists a \in \mathbb{Z}, \alpha \equiv a(\bmod p)\right\}=E_{F} \cap\left(K^{*}\right)^{p} .
$$

As already noticed, $E_{F}^{\mathrm{Kum}}$ is a subgroup of this latter group. Now, let $\alpha \in E_{F}$ be such that $\alpha \equiv a(\bmod p)$ for some integer $a$. Then there exists $\epsilon \in \mu_{p-1}$ such that $\alpha \epsilon \in U_{K}^{(p-1)}$. But $N_{K / \mathbb{Q}_{p}}(\alpha \epsilon)=1$. Therefore $\alpha \epsilon \in U_{K}^{(p)}$. Thus $\alpha \in E_{F}^{\mathrm{Kum}}$.

Now, recall that $\left(U_{K}\right)^{p}=\mu_{p-1} U_{K}^{(p+1)}$. Thus

$$
E_{F}^{\mathrm{Kum}} \subset\left\{\alpha \in E_{F}: \log _{p}(\alpha) \equiv 0\left(\bmod \mathfrak{p}_{K}^{p}\right)\right\} .
$$

Let $\alpha$ be in the right side group. Then, by Proposition 2.6, $\varphi_{k}(\alpha)=0$ for $k=1, \ldots, p-2$. Therefore $\alpha \in \mu_{p-1} U_{K}^{(p-1)}$. But $N_{K / \mathbb{Q}_{p}}(\alpha)=1$, thus $\alpha \in \mu_{p-1} U_{K}^{(p)}$, i.e. $\alpha \in E_{F}^{\mathrm{Kum}}$.

We define the index of regularity of $F$ to be

$$
r(F)=\left|\left\{i: 1 \leq i \leq l-1, B_{i(p-1) / l} \not \equiv 0(\bmod p)\right\}\right| .
$$

The index of irregularity of $F$ is then

$$
i(F)=l-1-r(F) .
$$

We call $F$ regular if $i(F)=0$. Note that, in this case, $p$ does not divide $h_{F}$ (see [10], Theorem 5.24).

If $F=\mathbb{Q}\left(\zeta_{p}\right)^{+}$, then $i(F)=i(p)$, the index of irregularity of $p$.

Theorem 3.2. Let $F$ be a real subfield of $\mathbb{Q}\left(\zeta_{p}\right)$ with $[F: \mathbb{Q}]=l \geq 2$.

(i) If $i=p-1$ or if $i \not \equiv 0(\bmod (p-1) / l)$, then

$$
\frac{\mathrm{Cyc}_{F}}{\mathrm{Cyc}_{F}^{\mathrm{Kum}}}(i)=0 .
$$

(ii) For $j=1, \ldots, l-1$,

$$
\frac{\operatorname{Cyc}_{F}}{\operatorname{Cyc}_{F}^{K u m}}\left(j \frac{(p-1)}{l}\right)=0 \Leftrightarrow B_{j(p-1) / l} \equiv 0(\bmod p) .
$$

(iii) We have

$$
\operatorname{dim}_{\mathbb{F}_{p}} \frac{\mathrm{Cyc}_{F}}{\mathrm{Cyc}_{F}^{\mathrm{Kum}}}=r(F) .
$$


Proof. We view $\mathrm{Cyc}_{F} / \mathrm{Cyc}_{F}^{\mathrm{Kum}}$ as an $\mathbb{F}_{p}[G]$-submodule of $\mathcal{U}$. Since $N_{K / \mathbb{Q}_{p}}\left(E_{F}\right)=\{1\}$, we have

$$
\frac{\mathrm{Cyc}_{F}}{\mathrm{Cyc}_{F}^{\mathrm{Kum}}}(p-1)=0 .
$$

Now, suppose that there exists $\epsilon \in E_{F}$ such that $\varphi_{i}(\epsilon) \neq 0$. Then

$$
\varphi_{i}\left(\epsilon^{(p-1) / l}\right)=\varphi_{i}\left(N_{K / \widehat{F}}(\epsilon)\right) \neq 0 .
$$

$\operatorname{But} \operatorname{Gal}(K / \widehat{F})=G^{l}$, thus

$$
\varphi_{i}\left(N_{K / \widehat{F}}(\epsilon)\right)=\frac{1}{l}\left(\sum_{\sigma \in G} \omega(\sigma)^{i l}\right) \varphi_{i}(\epsilon) .
$$

Thus $i l \equiv 0(\bmod p-1)$ and we get $(\mathrm{i})$.

By Proposition 2.5, for $k \geq 2$, we have

$$
\varphi_{k}\left(\frac{\sigma_{a}\left(\lambda_{p}\right)}{\lambda_{p}}\right)=(-1)^{k}\left(\omega\left(\sigma_{a}\right)^{k}-1\right) \frac{B_{k}}{k !} .
$$

Therefore we get (ii) and (iii).

We recover Kummer's Lemma:

Corollary 3.3. Suppose that $F$ is regular. Then $E_{F}^{\mathrm{Kum}}=\left(E_{F}\right)^{p}$.

Proof. In this case, we have

$$
\operatorname{dim}_{\mathbb{F}_{p}} \frac{\mathrm{Cyc}_{F}}{\mathrm{Cyc}_{F}^{\mathrm{Kum}}}=l-1 .
$$

But $\mathrm{Cyc}_{F} \cap E_{F}^{\mathrm{Kum}}=\mathrm{Cyc}_{F}^{\mathrm{Kum}}$, thus

Note that $\left(E_{F}\right)^{p} \subset E_{F}^{\mathrm{Kum}}$ and

$$
\operatorname{dim}_{\mathbb{F}_{p}} \frac{E_{F}}{E_{F}^{\text {Kum }}} \geq l-1 .
$$

$$
\operatorname{dim}_{\mathbb{F}_{p}} \frac{E_{F}}{\left(E_{F}\right)^{p}}=l-1 .
$$

Therefore we get the desired result.

A natural problem arises: do we have an equivalence in Kummer's Lemma? It is not difficult to show that if $p$ does not divide $h_{F}$, then $E_{F}^{\mathrm{Kum}}=$ $\left(E_{F}\right)^{p}$ implies that $F$ is regular. In fact, we have

Proposition 3.4. Let $F$ be a real subfield of $\mathbb{Q}\left(\zeta_{p}\right)$. Suppose that $p^{\max (i(F), 1)}$ does not divide $h_{F}$. Then $E_{F}^{\mathrm{Kum}}=\left(E_{F}\right)^{p}$ implies $i(F)=0$.

Proof. If $E_{F}^{\mathrm{Kum}}=\left(E_{F}\right)^{p}$, then

$$
\operatorname{dim}_{\mathbb{F}_{p}} \frac{E_{F}}{\mathrm{Cyc}_{F} E_{F}^{\mathrm{Kum}}}=i(F) .
$$

Since $h_{F}=\left(E_{F}: \mathrm{Cyc}_{F}\right), p^{i(F)}$ divides $h_{F}$. 
4. The orthogonal of local units. Recall that

$$
\mathcal{V}=\frac{\mathbb{Q}\left(\zeta_{p}\right) \cap U_{K}}{\mathbb{Q}\left(\zeta_{p}\right) \cap \mu_{p-1} U_{K}^{(p)}}
$$

is an $\mathbb{F}_{p}[G]$-module which is isomorphic to $\mathcal{U}=U_{K} /\left(\mu_{p-1} U_{K}^{(p)}\right)$. Let $\alpha \in$ $\mathbb{Q}\left(\zeta_{p}\right) \cap \mu_{p-1} U_{K}^{(p)}$. Then for every $\beta \in \mathbb{Q}\left(\zeta_{p}\right) \cap U_{K}$, we have $(\beta, \alpha)=1$. Therefore, if $B$ is a subgroup of $\mathcal{V}$, we set

$$
B^{\perp}=\{\alpha \in V: \forall b \in B,(b, \alpha)=(\alpha, b)=1\} .
$$

Via our isomorphism $\phi: \mathcal{V} \rightarrow \mathcal{U}$, we have an isomorphism

$$
B^{\perp} \equiv\{\alpha \in \mathcal{U}: \forall b \in B,(\alpha, \phi(b))=1\} .
$$

Note that, if $B$ is an $\mathbb{F}_{p}[G]$-submodule of $\mathcal{V}$, the above isomorphism is an isomorphism of $\mathbb{F}_{p}[G]$-modules.

Now, $\mathfrak{p}_{K}$ can be viewed as a $\mathbb{Z}_{p}$-submodule of $(D)_{L}$ (see Section 2). Since $[p]_{L}\left(\mathfrak{p}_{k}\right) \subset \mathfrak{p}_{K}^{p}$ and, for all $a \in \mathbb{Z}_{p},[a]_{L}\left(\mathfrak{p}_{K}^{p}\right) \subset \mathfrak{p}_{K}^{p}$, it follows that $\left(\mathfrak{p}_{K}\right)_{L} /\left(\mathfrak{p}_{K}^{p}\right)_{L}$ is an $\mathbb{F}_{p}$-vector space. Furthermore, since $F_{L}(X, Y) \equiv X+Y$ $(\bmod \operatorname{deg} p)$ and $[a]_{L} \equiv a X(\bmod \operatorname{deg} p)$ for all $a \in \mathbb{Z}_{p},\left(\mathfrak{p}_{K}\right)_{L} /\left(\mathfrak{p}_{K}^{p}\right)_{L}$ is the same as the usual $\mathbb{F}_{p}$-vector space $\mathfrak{p}_{K} / \mathfrak{p}_{K}^{p}$. Therefore we have an isomorphism of $\mathbb{F}_{p}[G]$-modules $\psi: \mathcal{U} \rightarrow \mathfrak{p}_{K} / \mathfrak{p}_{K}^{p}, u \mapsto g_{p}(u-1)$. But recall that

$$
\forall u \in U_{K}^{(1)}, \forall \alpha \in K^{*}, \quad f_{p}\left(\left\langle g_{p}(u-1), \alpha\right\rangle_{L}\right)=(u, \alpha)-1 .
$$

We deduce from the above discussion that $B^{\perp}$ is isomorphic to the $\mathbb{F}_{p}$-vector space

$$
\left\{z \in \mathfrak{p}_{K} / \mathfrak{p}_{K}^{p}:\langle z, B\rangle_{L}=0\right\} .
$$

Theorem 4.1. Let $B$ be an $\mathbb{F}_{p}[G]$-submodule of $\mathcal{V}$. Then, for $1 \leq i \leq$ $p-1$, we have

$$
\operatorname{dim}_{\mathbb{F}_{p}} B^{\perp}(i)+\operatorname{dim}_{\mathbb{F}_{p}} B(p-i)=1 .
$$

Proof. First note that $B^{\perp}$ is an $\mathbb{F}_{p}[G]$-submodule of $\mathcal{V}$. Now, we identify $B^{\perp}$ and $\left\{z \in \mathfrak{p}_{K} / \mathfrak{p}_{K}^{p}:\langle z, B\rangle_{L}=0\right\}$ which is an $\mathbb{F}_{p}[G]$-submodule of $\mathfrak{p}_{K} / \mathfrak{p}_{K}^{p}$. Note that $\mathfrak{p}_{K} / \mathfrak{p}_{K}^{p}$ is an $\mathbb{F}_{p}$-vector space of dimension $p-1$ with $\left\{\lambda_{L}, \ldots, \lambda_{L}^{p-1}\right\}$ as a base over $\mathbb{F}_{p}$.

For simplification, we set $e_{i}=e_{\omega^{i}}$ for $i=1, \ldots, p-1$. Let $j$ be an integer, $1 \leq j \leq p-1$. We have:

- $e_{i} \lambda_{L}^{j}=0$ if $j \neq i$,

- $e_{i} \lambda_{L}^{j}=\lambda_{L}^{j}$ if $j=i$.

Therefore

$$
\frac{\mathfrak{p}_{K}}{\mathfrak{p}_{K}^{p}}(i)=\mathbb{F}_{p} \lambda_{L}^{i}
$$

This implies that

$$
B^{\perp}(i) \neq 0 \Leftrightarrow \lambda_{L}^{i} \in B^{\perp} .
$$


Now, let $2 \leq j \leq p-1,1 \leq i \leq p-1$. Let $b \in B$. By Theorem 2.7, we have

$$
\left\langle\lambda_{L}^{j}, e_{i} b\right\rangle_{L}=\left[\varphi_{p-j}\left(e_{i} b\right)\right]_{L}\left(\lambda_{L}\right) .
$$

But $\varphi_{p-j}\left(e_{i} b\right)=0$ if $p-j \neq i$ and $\varphi_{p-j}\left(e_{i} b\right)=\varphi_{i}(b)$ if $i=p-j$. Now, note that

$$
\lambda_{L}^{j} \in B^{\perp} \Leftrightarrow \forall i, 1 \leq i \leq p-1,\left\langle\lambda_{L}^{j}, B(i)\right\rangle_{L}=0 .
$$

Furthermore

$$
\forall b \in B, \quad\left\langle\lambda_{L}, b\right\rangle_{L}=\left[\frac{N_{K / \mathbb{Q}_{p}}\left(u^{-1}\right)-1}{p}\right]_{L}\left(\lambda_{L}\right) .
$$

Thus $\lambda_{L} \in B^{\perp} \Leftrightarrow B(p-1)=0$. The theorem follows.

Corollary 4.2. Let $B$ be an $\mathbb{F}_{p}[G]$-submodule of $\mathcal{V}$. Then

$$
\operatorname{dim}_{\mathbb{F}_{p}} B^{\perp}+\operatorname{dim}_{\mathbb{F}_{p}} B=p-1 .
$$

Corollary 4.3. Let $B$ be an $\mathbb{F}_{p}[G]$-submodule of $\mathcal{V}$. Then

$$
\left(B^{\perp}\right)^{\perp}=B \text {. }
$$

Proof. Note that $B^{\perp}$ is an $\mathbb{F}_{p}[G]$-submodule of $\mathcal{V}$. Thus, by Corollary 4.2,

$$
\operatorname{dim}_{\mathbb{F}_{p}}\left(B^{\perp}\right)^{\perp}+\operatorname{dim}_{\mathbb{F}_{p}} B^{\perp}=p-1 .
$$

But $B \subset\left(B^{\perp}\right)^{\perp}$, and by Corollary 4.2 ,

$$
\operatorname{dim}_{\mathbb{F}_{p}} B+\operatorname{dim}_{\mathbb{F}_{p}} B^{\perp}=p-1 .
$$

Thus $B=\left(B^{\perp}\right)^{\perp}$.

Now, let $F$ be a real subfield of $\mathbb{Q}\left(\zeta_{p}\right)$ with $[F: \mathbb{Q}]=l \geq 2$. If we apply Theorems 3.2 and 4.1, we get

Theorem 4.4. (i) Let $i$ be an integer, $1 \leq i \leq p-1$. Then

$$
\operatorname{dim}_{\mathbb{F}_{p}} \operatorname{Cyc}_{F}^{\perp}(i)+\operatorname{dim}_{\mathbb{F}_{p}} \frac{\mathrm{Cyc}_{F}}{\operatorname{Cyc}_{F}^{\mathrm{Kum}}}(p-i)=1 .
$$

Thus $\mathrm{Cyc}_{F}^{\perp} \neq 0$ if and only if $i \not \equiv 1(\bmod (p-1) / l), i=p-1$, or $i \equiv 1$ $(\bmod (p-1) / l)$ and $B_{p-i} \equiv 0(\bmod p)$. In particular,

$$
\operatorname{dim}_{\mathbb{F}_{p}} \operatorname{Cyc}_{F}^{\perp}=p-1-r(F) .
$$

(ii) Let $i$ be an integer, $1 \leq i \leq p-1$. Then

$$
\operatorname{dim}_{\mathbb{F}_{p}} \frac{\mathrm{Cyc}_{F}^{\perp}}{E_{F}^{\perp}}(i)=\operatorname{dim}_{\mathbb{F}_{p}} \frac{E_{F}}{\mathrm{Cyc}_{F} E_{F}^{\mathrm{Kum}}}(p-i) .
$$

Let $I$ be the Stickelberger ideal (see [10], Chapter 6) and let $\mathcal{I}$ be its image in $\mathbb{F}_{p}[G]$. Let $F=\mathbb{Q}\left(\zeta_{p}\right)^{+}$. Then, by Theorem 4.4 and [10], Section 6.3, 
there exists a surjective morphism of $\mathbb{F}_{p}[G]$-modules

$$
\frac{\mathbb{F}_{p}[G]^{-}}{\mathcal{I}^{-}} \rightarrow \frac{\mathrm{Cyc}_{F}^{\perp}}{E_{F}^{\perp}} .
$$

Since $\operatorname{dim}_{\mathbb{F}_{p}} \mathbb{F}_{p}[G]^{-} / \mathcal{I}^{-}=i(p)$, this morphism is an isomorphism if and only if $E_{F}^{\mathrm{Kum}}=\left(E_{F}\right)^{p}$.

5. Mirimanoff's polynomials. In his attempt to prove the first case of Fermat's Last Theorem, D. Mirimanoff introduced the polynomials

$$
M_{k}(X)=\sum_{i=1}^{p-1} i^{k-1} X^{i} \in \mathbb{F}_{p}[X], \quad k \geq 1 \text { an integer. }
$$

Note that $(X-1) M_{1}(X)=X^{p}-X$. Let $\Gamma=X \frac{d}{d X}$. Then, for $k \geq 1$, we have

$$
\Gamma^{k} M_{1}=M_{k+1} .
$$

From this relation, we deduce immediately that, for $2 \leq k \leq p-1$, we have

$$
M_{k}(X)=X(X-1)^{p-k} P_{k}(X),
$$

where $P_{k}(X) \in \mathbb{F}_{p}[X]$ is of degree $k-2$ and $P_{k}(0) \not \equiv 0(\bmod p), P_{k}(1) \not \equiv 0$ $(\bmod p)$.

Note that, if $k$ is odd, $3 \leq k \leq p-2$, we have (see [5], Chapter 8):

$$
M_{k}(X)=(-1)^{k} X(X+1)(X-1)^{p-k} L_{k}(-X),
$$

where $L_{k}(X) \in \mathbb{F}_{p}[X]$ is of degree $k-3$. The first polynomials $L_{k}(X)$ are:

$$
\begin{aligned}
& L_{3}(X)=1, \\
& L_{5}(X)=X^{2}-10 X+1, \\
& L_{7}(X)=X^{4}-56 X^{3}+246 X^{2}-56 X+1, \\
& L_{9}(X)=X^{6}-246 X^{5}+4047 X^{4}-11572 X^{3}+4047 X^{2}-246 X+1 .
\end{aligned}
$$

In this section, we will relate the study of the non-trivial zeros in $\mathbb{F}_{p}^{*}$ of the polynomials $M_{k}(X), k$ odd, to the orthogonal of cyclotomic units.

Note that the number of $k$ even, $2 \leq k \leq p-3$, such that $-1 \in \mathbb{F}_{p}^{*}$ is a root of $M_{k}(X)$ is connected to $i(p)$ :

Lemma 5.1. (i) Let $k$ be an even integer, $2 \leq k \leq p-3$. Then

$$
M_{k}(-1) \equiv 2\left(2^{k}-1\right) \frac{B_{k}}{k}(\bmod p) .
$$

(ii) $M_{p-1}(-1) \equiv \frac{2^{p}-2}{p}(\bmod p)$.

Proof. (i) is a consequence of Proposition 2.5; for (ii) see [5], Chapter 8. 
Recall that we identify $\mathcal{V}$ and $\mathcal{U}$. Set

$$
\varepsilon_{+}=\sum_{i \equiv 0(\bmod 2)} e_{\omega^{i}} \in \mathbb{F}_{p}[G] \text { and } \varepsilon_{-}=\sum_{i \equiv 1(\bmod 2)} e_{\omega^{i}} \in \mathbb{F}_{p}[G] .
$$

Then $\varepsilon_{+} \varepsilon_{-}=0, \varepsilon_{+}^{2}=\varepsilon_{+}, \varepsilon_{-}^{2}=\varepsilon_{-}, 1=\varepsilon_{+}+\varepsilon_{-}, \sigma_{-1} \varepsilon_{+}=\varepsilon_{+}$and $\sigma_{-1} \varepsilon_{-}=-\varepsilon_{-}$. We set $\mathcal{V}^{+}=\varepsilon_{+} \mathcal{V}$ and $\mathcal{V}^{-}=\varepsilon_{-} \mathcal{V}$. Then

$$
\mathcal{V}^{+}=\bigoplus_{i \equiv 0(\bmod 2)} \mathcal{V}(i), \quad \mathcal{V}^{-}=\bigoplus_{i \equiv 1(\bmod 2)} \mathcal{V}(i)
$$

Furthermore

$$
\operatorname{dim}_{\mathbb{F}_{p}} \mathcal{V}^{+}=\operatorname{dim}_{\mathbb{F}_{p}} \mathcal{V}^{-}=(p-1) / 2
$$

Note also that

$$
\mathcal{V}^{+}=\frac{\mathbb{Q}\left(\zeta_{p}\right)^{+} \cap U_{K}}{\mathbb{Q}\left(\zeta_{p}\right)^{+} \cap \mu_{p-1} U_{K}^{(p)}} .
$$

Let $\epsilon \in \mu_{p-1}$. We set

$$
\varrho_{\epsilon}=\frac{\epsilon-\zeta_{p}}{\epsilon-\zeta_{p}^{-1}} .
$$

Then $\varrho_{\epsilon} \in \mathcal{V}^{-}$. In this section, we suppose that $p \geq 5$.

Lemma 5.2. $\mathcal{V}^{-}$is generated as $\mathbb{F}_{p}[G]$-module by the $\varrho_{\epsilon}, \epsilon \in \mu_{p-1} \backslash$ $\{1,-1\}$.

Proof. Let $\epsilon \in \mu_{p-1}, \epsilon \neq 1$. Then, by Proposition 2.5, we have $\varphi_{1}\left(\varrho_{\epsilon}\right)$ $\neq 0$. Thus

$$
\mathcal{V}^{-}(1)=\mathbb{F}_{p} e_{\omega} \varrho_{\epsilon} .
$$

Let $k$ be an odd integer, $3 \leq k \leq p-2$. By Proposition 2.5, we have

$$
\mathcal{V}^{-}(k)=\mathbb{F}_{p} e_{\omega^{k}} \varrho_{\epsilon} \Leftrightarrow \varphi_{k}\left(\varrho_{\epsilon}\right) \neq 0 \Leftrightarrow M_{k}(\epsilon) \not \equiv 0(\bmod p) .
$$

But there exists $\epsilon \in \mu_{p-1} \backslash\{1,-1\}$ such that $M_{k}(\epsilon) \not \equiv 0(\bmod p)$. The lemma follows.

Lemma 5.3. Let $F$ be a real subfield of $\mathbb{Q}\left(\zeta_{p}\right)$ with $[F: \mathbb{Q}]=l \geq 2$. Then $\varrho_{\epsilon} \in \mathrm{Cyc}_{F}^{\perp}$ if and only if for $j=1, \ldots, l-1$,

$$
B_{j(p-1) / l} M_{p-j(p-1) / l}(\epsilon) \equiv 0(\bmod p) .
$$

Proof. By the proof of Proposition 2.6, we have

$$
g_{p}\left(\varrho_{\epsilon}-1\right) \equiv \sum_{k=1}^{p-2} \frac{1}{k} \varphi_{k}\left(\varrho_{\epsilon}\right) \lambda_{L}^{k}\left(\bmod \mathfrak{p}_{K}^{p}\right) .
$$

Thus, by Theorem 2.7, Proposition 2.5 and Theorem 3.2, if

$$
B_{j(p-1) / l} M_{p-i(p-1) / l}(\epsilon) \equiv 0(\bmod p) \quad \text { for } j=1, \ldots, l-1,
$$

then $\varrho_{\epsilon} \in \mathrm{Cyc}_{F}^{\perp}$. 
Conversely, assume that $\varrho_{\epsilon} \in \mathrm{Cyc}_{F}^{\perp}$. Let $B$ be the $\mathbb{F}_{p}[G]$-submodule of $\mathcal{V}^{-}$generated by $\varrho_{\epsilon}$. By Theorem 4.1, we have

$$
\operatorname{dim}_{\mathbb{F}_{p}} B(i)+\operatorname{dim}_{\mathbb{F}_{p}} \frac{\mathrm{Cyc}_{F}}{\mathrm{Cyc}_{F}^{\mathrm{Kum}}}(p-1) \leq 1 .
$$

It remains to apply Proposition 2.5 and Theorem 3.2.

G. Terjanian has conjectured (see [8]) that for every odd prime number, $\varrho_{\epsilon} \in \mathrm{Cyc}_{F}^{\perp} \Rightarrow \epsilon=1$ or $\epsilon=-1$, where $F=\mathbb{Q}\left(\zeta_{p}\right)^{+}$. By Lemma 5.3, Terjanian's conjecture is equivalent to the statement that the Kummer system of congruences

$$
B_{2 j} M_{p-2 j} \equiv 0(\bmod p), \quad 1 \leq j \leq(p-3) / 2,
$$

has only the trivial solutions, i.e. 0,1 and -1 . L. Skula has proved (see [7]) that if Terjanian's conjecture is false for a prime $p$ then $i(p) \geq[\sqrt[3]{p / 2}]$.

Theorem 5.4. Let $x, y \in \mathbb{Z}$ be such that $x y\left(x^{2}-y^{2}\right) \not \equiv 0(\bmod p)$. Let $B$ be the $\mathbb{F}_{p}[G]$-submodule of $\mathcal{V}$ generated by $x+y \zeta_{p}$. Then

$$
\operatorname{dim}_{\mathbb{F}_{p}} B^{-} \geq \sqrt{p}-1 \text {. }
$$

Proof. Suppose that $\operatorname{dim}_{\mathbb{F}_{p}} B^{-}<\sqrt{p}-1$. Set $r=[\sqrt{p}]-1$. Note that $\zeta_{p} \in B^{-}$. Consider the set of all products

$$
\zeta_{p}^{b_{0}} \prod_{i=1}^{r}\left(x+y \zeta_{p}^{i}\right)^{b_{i}}
$$

where $0 \leq b_{i}<p$ for $i=0, \ldots, r$. The number of such products is $p^{r+1}>$ $\left|B^{-}\right|$. Therefore, two of them must agree in their $B^{-}$-components, so we may divide and obtain

$$
\prod_{i=1}^{r}\left(x+y \zeta_{p}^{i}\right)^{a_{i}} \equiv \zeta_{p}^{\nu} \delta(\bmod p),
$$

where $-p<a_{i}<p$ and some $a_{i}$ are non-zero (because a non-trivial power of $\zeta_{p}$ is not congruent to a real number modulo $\left.p\right), \delta \in \mathbb{Q}\left(\zeta_{p}\right)^{+}$and $\nu \geq 0$. Thus, we get

$$
\prod_{i=1}^{r} \frac{\left(x+y \zeta_{p}^{i}\right)^{a_{i}}}{\left(y+x \zeta_{p}^{i}\right)^{a_{i}}} \equiv \zeta_{p}^{v}(\bmod p)
$$

for some $v \geq 0$. But, by the proof of Eichler's Theorem (see [10], Theorem 6.23), this implies that $x y\left(x^{2}-y^{2}\right) \equiv 0(\bmod p)$, a contradiction.

COROLlary 5.5. Let $p \geq 5$ be a prime number. If Terjanian's conjecture is false for the prime $p$, then:

(i) $2^{p-1} \equiv 1\left(\bmod p^{2}\right)$;

(ii) $B_{p-3} \equiv 0(\bmod p)$;

(iii) $i(p) \geq \sqrt{p}-2$. 
Proof. Let $C$ be the group of cyclotomic units of $\mathbb{Q}\left(\zeta_{p}\right)$ and let $F=$ $\mathbb{Q}\left(\zeta_{p}\right)^{+}$. Then $\epsilon-\zeta_{p}$ is orthogonal to $C$ for the norm residue symbol if and only if $\varrho_{\epsilon} \in \mathrm{Cyc}_{F}^{\perp}$ (see [2]). Therefore (i) and (ii) are a consequence of [8], Enoncé 8. Now, (iii) is a consequence of Theorem 5.4, Lemma 5.3 and Proposition 2.5.

Note that the $A B C$ conjecture implies that Terjanian's conjecture is true for infinitely many primes $p$ (see [6]). It would be interesting to find analogues of Terjanian's conjecture for real subfields of $\mathbb{Q}\left(\zeta_{p}\right)$ (see [1]).

6. $p$-adic regulators and Kummer subgroups of units. Let $F$ be a real subfield of $\mathbb{Q}\left(\zeta_{p}\right)$ with $[F: \mathbb{Q}]=l, l \geq 2$. We set $G_{F}=\operatorname{Gal}\left(\widehat{F} / \mathbb{Q}_{p}\right)$ and $\chi=\omega^{(p-1) / l}$. Then

$$
\widehat{G}_{F}=\langle\chi\rangle \text {. }
$$

We denote the $p$-adic regulator of $F$ by $R_{p}(F)$ and the discriminant of $F$ by $d(F)$. Let $\varepsilon \in E_{F}$; we denote by $A_{\varepsilon}$ the subgroup of $E_{F}$ generated by -1 and $\sigma(\varepsilon), \sigma \in G_{F}$. We say that $\varepsilon$ is a Minkowski unit if $A_{\varepsilon}$ is of finite index in $E_{F}$.

Proposition 6.1. Let $\varepsilon \in E_{F}$ be a Minkowski unit. Then

$$
\left(E_{F}: A_{\varepsilon}\right) \frac{R_{p}(F)}{\sqrt{d(F)}} \equiv \pm \frac{l^{2(l-1)}}{(l-1) !} \prod_{k=1}^{l-1} \varphi_{k(p-1) / l}(\varepsilon)(\bmod p) .
$$

Proof. Let $\varepsilon$ be a Minkowski unit. Set

$$
R_{p}\left(A_{\varepsilon}\right)=\operatorname{det}\left(\log _{p}(\sigma \tau(\varepsilon))\right)_{\sigma, \tau \in G_{F} \backslash\{1\}} .
$$

Then $R_{p}\left(A_{\varepsilon}\right) \neq 0$ and (see [10], Lemma 4.15)

$$
\left(E_{F}: A_{\varepsilon}\right)= \pm \frac{R_{p}\left(A_{\varepsilon}\right)}{R_{p}(F)} .
$$

But, from [10], Lemma 5.26,

$$
R_{p}\left(A_{\varepsilon}\right)=\prod_{j=1}^{l-1}\left(\sum_{\sigma \in G_{F}} \chi(\sigma)^{-j} \log _{p}(\sigma(\varepsilon))\right) .
$$

Now, by Proposition 2.6,

$$
\log _{p}(\sigma(\varepsilon)) \equiv \sum_{j=1}^{l-1} \frac{1}{j(p-1) / l} \chi(\sigma)^{-j} \varphi_{j(p-1) / l}(\varepsilon) \lambda_{L}^{j(p-1) / l}\left(\bmod \mathfrak{p}_{K}^{p}\right) .
$$

Thus, we have

$$
\sum_{\sigma \in G_{F}} \chi(\sigma)^{-k} \log _{p}(\sigma(\varepsilon)) \equiv \frac{l^{2}}{k(p-1)} \varphi_{k(p-1) / l}(\varepsilon) \lambda_{L}^{k(p-1) / l}\left(\bmod \mathfrak{p}_{K}^{p}\right) .
$$


Therefore, there exists $a_{k} \in \mathbb{Z}_{p}, a_{k} \equiv \varphi_{k(p-1) / l}(\varepsilon)$, such that

$$
\sum_{\sigma \in G_{F}} \chi(\sigma)^{-k} \log _{p}(\sigma(\varepsilon))=\lambda_{L}^{k(p-1) / l}\left(\frac{l^{2}}{k(p-1)} a_{k}+u_{k}\right),
$$

where $u_{k} \in \mathfrak{p}_{K}^{1+(p-1) / l}$. We get

$$
R_{p}\left(A_{\varepsilon}\right)=\lambda_{L}^{(p-1)(l-1) / 2} \prod_{k=1}^{l-1}\left(\frac{l^{2}}{k(p-1)} a_{k}+u_{k}\right) .
$$

But $\sqrt{d(F)}= \pm \lambda_{L}^{(p-1)(l-1) / 2}$. Therefore

$$
\left(E_{F}: A_{\varepsilon}\right) \frac{R_{p}(F)}{\sqrt{d(F)}} \equiv \pm \frac{l^{2(l-1)}}{(l-1) !} \prod_{k=1}^{l-1} \varphi_{k(p-1) / l}(\varepsilon)\left(\bmod \mathfrak{p}_{K}^{1+(p-1) / l}\right) .
$$

But, since $R_{p}(F) / \sqrt{d(F)} \in \mathbb{Z}_{p}$, this congruence holds modulo $p$.

Corollary 6.2. Let $\varepsilon$ be a Minkowski unit, $\varepsilon \in E_{F}$. Then

$$
(2 l)^{l-1} h_{F} \prod_{k=1}^{l-1} \varphi_{k(p-1) / l}(\varepsilon) \equiv \pm\left(E_{F}: A_{\varepsilon}\right) \prod_{k=1}^{l-1} B_{k(p-1) / l}(\bmod p) .
$$

Proof. By [10], Theorem 5.24,

$$
2^{l-1} h_{F} \frac{R_{p}(F)}{\sqrt{d(F)}}=\prod_{j=1}^{l-1} L_{p}\left(1, \chi^{j}\right) .
$$

Now

$$
L_{p}\left(1, \chi^{j}\right) \equiv \frac{l}{j} B_{j(p-1) / l}(\bmod p)
$$

Therefore

$$
2^{l-1} h_{F} \frac{R_{p}(F)}{\sqrt{d(F)}} \equiv \frac{l^{l-1}}{(l-1) !} \prod_{j=1}^{l-1} B_{j(p-1) / l}(\bmod p) .
$$

Let $\varepsilon$ be a Minkowski unit. By Proposition 6.1, we have

$$
\left(E_{F}: A_{\varepsilon}\right) \frac{R_{p}(F)}{\sqrt{d(F)}} \equiv \pm \frac{l^{2(l-1)}}{(l-1) !} \prod_{j=1}^{l-1} \varphi_{j(p-1) / l}(\varepsilon)(\bmod p) .
$$

The corollary follows.

Let $\varepsilon_{1}, \ldots, \varepsilon_{l-1}$ be a system of fundamental units of $F$. We set

$$
R_{F} \equiv\left(\operatorname{det}\left(\frac{1}{j(p-1) / l} \varphi_{j(p-1) / l}\left(\varepsilon_{i}\right)\right)_{1 \leq i, j \leq l-1}\right)^{2}(\bmod p) .
$$

Note that $R_{F}$ modulo $p$ is independent of the choice of $\varepsilon_{1}, \ldots, \varepsilon_{l-1}$ (see [4]). 
LEMMA 6.3. $R_{F} \not \equiv 0(\bmod p)$ if and only if $E_{F}^{\mathrm{Kum}}=\left(E_{F}\right)^{p}$.

Proof. It is clear that if $R_{F} \not \equiv 0(\bmod p)$ then $E_{F}^{\mathrm{Kum}}=\left(E_{F}\right)^{p}$.

Conversely, assume that $E_{F}^{\mathrm{Kum}}=\left(E_{F}\right)^{p}$. Let $\varepsilon$ be a generator of the cyclic $\mathbb{F}_{p}\left[G_{F}\right]$-module $E_{F} / E_{F}^{\mathrm{Kum}}$. Set

$$
B \equiv\left(\operatorname{det}\left(\frac{1}{j(p-1) / l} \varphi_{j(p-1) / l}(\sigma(\varepsilon))\right)_{1 \leq j \leq l-1, \sigma \in G_{F} \backslash\{1\}}\right)^{2}(\bmod p) .
$$

The rank of this latter matrix is equal to the rank of

$$
\left(\chi(\sigma)^{j}\right)_{1 \leq j \leq l-1, \sigma \in G_{F} \backslash\{1\}} .
$$

Therefore $B \not \equiv 0(\bmod p)$. By Proposition 2.6 and [4], page 113,

$$
B \equiv\left(E_{F}: A_{\varepsilon}\right)^{2} R_{F}(\bmod p) .
$$

Therefore $R_{F} \not \equiv 0(\bmod p)$.

If we apply Proposition 2.6, by the proof of [4], Theorem 1A, we get

THEOREM 6.4. Let $g$ be a primitive root modulo $p$. We have $4^{l-1} h_{F}^{2} R_{F}$

$$
\equiv \frac{l^{2}}{(l-1) !^{2}}\left(\operatorname{det}\left(g^{(p-1)(i-1) k / l}\right)_{1 \leq i, k \leq l-1}\right)^{2} \prod_{j=1}^{l-1} \frac{B_{j(p-1) / l}^{2}}{((j(p-1) / l) !)^{2}}(\bmod p) .
$$

TheOREM 6.5 .

$$
E_{F}^{\mathrm{Kum}}=\left(E_{F}\right)^{p} \quad \text { if and only if } \frac{R_{p}(F)}{\sqrt{d(F)}} \not \equiv 0(\bmod p) .
$$

Proof. Let $\varepsilon_{1}, \ldots, \varepsilon_{l-1}$ be a system of fundamental units of $F$. Set $\beta_{i}=$ $\log _{p}\left(\varepsilon_{i}\right)$ for $i=1, \ldots, l-1$ and $\beta_{l}=1$ (recall that $l=[F: \mathbb{Q}]$ ). We have $\widehat{F}=\mathbb{Q}_{p}\left(\lambda_{L}^{(p-1) / l}\right)$. Thus

$$
O_{\widehat{F}}=\bigoplus_{j=0}^{l-1} \mathbb{Z}_{p} \lambda_{L}^{j(p-1) / l}
$$

Therefore, for $i=1, \ldots, l$, we can write

$$
\beta_{i}=\sum_{j=0}^{l-1} a_{i j} \lambda_{L}^{j(p-1) / l}
$$

where $a_{i j} \in \mathbb{Z}_{p}$. But

$$
\operatorname{det}\left(\sigma\left(\beta_{i}\right)\right)_{\sigma \in \operatorname{Gal}\left(\widehat{F} / \mathbb{Q}_{p}\right), i=1, \ldots, l}=l R_{p}(F) .
$$

Furthermore

$$
\operatorname{det}\left(\sigma\left(\beta_{i}\right)\right)=\operatorname{det}\left(a_{i j}\right) \operatorname{det}\left(\sigma\left(\lambda_{L}^{j(p-1) / l}\right)\right) \text {. }
$$


But, for $i=1, \ldots, l-1$, we have

$$
a_{i j} \equiv-\frac{l}{j} \varphi_{j(p-1) / l}\left(\varepsilon_{i}\right)(\bmod p)
$$

for $j=1, \ldots, l-1$ and $a_{i 0} \equiv 0(\bmod p)$. Therefore

$$
\operatorname{det}\left(a_{i j}\right)^{2} \equiv R_{F}(\bmod p) \text {. }
$$

The theorem follows.

\section{References}

[1] C. Helou, Norm residue symbol and cyclotomic units, Acta Arith. 73 (1995), 147188.

[2] - Proof of a conjecture of Terjanian for regular primes, C. R. Math. Rep. Acad. Sci. Canada 18 (1996), no. 5, 193-198.

[3] S. Lang, Cyclotomic Fields I and II, Springer, 1990.

[4] T. Metsänkylä, A class number congruence for cyclotomic fields and their subfields, Acta Arith. 23 (1973), 107-116.

[5] P. Ribenboim, 13 Lectures on Fermat's Last Theorem, Springer, 1979.

[6] J. Silverman, Wieferich's Criterion and the ABC conjecture, J. Number Theory 30 (1988), 226-237.

[7] L. Skula, The orders of solutions of the Kummer system of congruences, Trans. Amer. Math. Soc. 343 (1994), 587-607.

[8] G. Terjanian, Sur la loi de réciprocité des puissances l-èmes, Acta Arith. 54 (1989), $87-125$.

[9] S. V. Vostokov, Artin-Hasse exponentials and Bernoulli numbers, in: Amer. Math. Soc. Transl. (2) 166, Providence, RI, 1995, 149-156.

[10] L. C. Washington, Introduction to Cyclotomic Fields, Springer, 1997.

Laboratoire SDAD

Université de Caen

Campus II

BP 5186

Boulevard Maréchal Juin

14032 Caen Cedex, France

E-mail: angles@math.unicaen.fr

Received on 22.11.1999

and in revised form on 9.5.2000 